\title{
Philosophiques
}

\section{Czesław Miłosz, la liberté intérieure et la sottise}

\section{Kevin Mulligan}

Volume 40, numéro 1, printemps 2013

Littérature et connaissance

URI : https://id.erudit.org/iderudit/1018379ar

DOI : https://doi.org/10.7202/1018379ar

Aller au sommaire du numéro

Éditeur(s)

Société de philosophie du Québec

ISSN

0316-2923 (imprimé)

1492-1391 (numérique)

Découvrir la revue

Citer cet article

Mulligan, K. (2013). Czesław Miłosz, la liberté intérieure et la sottise. Philosophiques, 40(1), 107-120. https://doi.org/10.7202/1018379ar
Résumé de l'article

La philosophie des valeurs cognitives, et des vertus et des vices intellectuels, prête peu d'attention au phénomène de la liberté intérieure et de son rapport à la sottise. On doit au poète et penseur polonais, Czesław Miłosz, une étude classique de ce rapport, La pensée captive (1953), examinée ici. Il est aussi question ici de l'apport d'une autre analyse de Miłosz au sujet du coeur de Julien Sorel et du ressentiment à la philosophie de la sottise. d'utilisation que vous pouvez consulter en ligne.

https://apropos.erudit.org/fr/usagers/politique-dutilisation/ 


\title{
Czesław Miłosz, la liberté intérieure et la sottise
}

\author{
KEVIN MULLIGAN
}

Université de Genève

\begin{abstract}
RÉSUMÉ. - La philosophie des valeurs cognitives, et des vertus et des vices intellectuels, prête peu d'attention au phénomène de la liberté intérieure et de son rapport à la sottise. On doit au poète et penseur polonais, Czesław Miłosz, une étude classique de ce rapport, La pensée captive (1953), examinée ici. Il est aussi question ici de l'apport d'une autre analyse de Miłosz au sujet du cœur de Julien Sorel et du ressentiment à la philosophie de la sottise.
\end{abstract}

SUMMARY. - The philosophy of cognitive values and of intellectual vices and virtues pays little attention to the phenomenon of inner freedom and its relation to foolishness. We owe to the Polish poet and philosopher, Czesław Miłosz, a classic study of this relation, The Captive Mind (1953). I examine the contribution of this study and of an earlier and little known analysis by Miłosz of the heart of Julien Sorel and resentment to the philosophy of foolishness.

Qu'est-ce exactement qu'une pensée captive ou asservie, qu'une pensée qui n'est pas libre? La pensée, ou plus généralement l'esprit, peut-elle se trouver dans un état d'esclavage? Qui la met dans une telle position? Qui l'y maintient? Et comment? Un homme peut se trouver dans l'état d'être contraint par autrui et donc de n'être pas libre. Mais sa pensée peut-elle l'être? Y a-t-il une liberté intérieure qui se distingue de la liberté extérieure, par exemple politique? On a souvent répondu par l'affirmative. Dans le monde francophone, c'est principalement Pierre Hadot qui a exploré l'histoire de ces réponses. Mais c'est d'abord au sein de la tradition philosophique de l'Autriche-Hongrie que la philosophie de la liberté intérieure a trouvé son analyste, dans le beau livre de Heinrich Gomperz Die Lebensauffassung der griechischen Philosophen und das Ideal der inneren Freiheit ${ }^{1}$.

La liberté intérieure s'oppose à plusieurs phénomènes et a donc beaucoup de visages. Elle est sans doute incompatible avec l'étroitesse d'esprit et ses voisins le dogmatisme, l'insularité et la bigoterie ${ }^{2}$. Mais aussi avec le désir mimétique et la contagion affective, par exemple la contagion des convictions. Une variété particulière de l'esclavage intérieur est au centre du remarquable

1. Gomperz I9I 5 (1904, I927); Voelke I993; Hadot 2002, I995 p. ex., p. 339. Dans la philosophie autrichienne l'identification par Herbart de l'analyse des vertus que donne Platon dans le quatrième livre de la République avec «l'idée de liberté intérieure » fut reprise et développée par Wildauer I877 (\$I9-20). Max Scheler, comme d'autres penseurs allemands, aime dire des Anglais qu'ils ne connaissent que la liberté extérieure. Comme Max Weber, il reproche aussi à ses concitoyens d'ignorer ou de dévaluer la liberté extérieure au nom de l'Innerlichkeit. Sur l'histoire des conceptions allemandes de la liberté, cf. Krieger 1957.

2. Selon Max Scheler, l'étroitesse d'esprit (Borniertheit) et sa contrepartie morale, «cant» (l'un des deux vices que l'on appelle traditionnellement «le vice anglais»), sont deux formes de "geistige Unfreiheit» (Scheler, I982, p. 237). 
ouvrage de Czesław Miłosz, La pensée captive. Essai sur les logocraties populaires (1953), un ouvrage qui appartient lui aussi à la tradition de réflexion de l'Autriche-Hongrie. Miłosz essaie d'y montrer "comment l'esprit humain travaille dans les démocraties populaires» (p. I 5 ). Il y donne «une analyse descriptive» (p. I9) des hommes qui ne sont pas "intérieurement libres» (p. 2I4) ainsi que de "l'asservissement par la conscience» (p. 243), «une chose bien plus grave que la contrainte par la force matérielle» (p. 27) - analyse qui n’a jamais été dépassée.

Le but de ce qui suit est de cerner de plus près la variété de la pensée captive décrite par le poète et penseur polonais.

Le portrait de «A.» esquisse la Gesinnung de ce «moraliste»:

L'attitude d'A. était métaphysique et tragique; le problème de la pureté le tourmentait — de la pureté morale, et de la pureté du ton dans ce qu'il écrivait; [...] Ce besoin de pureté, d'une pureté non terrestre, dirais-je, était chez A. un trait foncier de caractère qui, dans ses relations avec les hommes, se traduisait par un abord hautain et guindé. [Il] voulait être, par son œuvre, une autorité morale (p. I I 8-9).

A., avec sa vision tragique du monde, cherchait [dans le catholicisme] [...] une draperie [...] Mais surtout, le langage catholique introduisait immédiatement le ton élevé, si nécessaire à A. Enfin celui-ci assouvissait de la sorte son goût pour la pourpre ecclésiastique (p. I20).

C'est un certain nombre de vices et d'autres incapacités qui, sur la base de cet arrière- plan, vont faire d'A. un exemple d'un type particulier de l'esclavage intérieur. D'abord, " occupé à construire des conflits intérieurs, il avait des yeux mal entraînés à l'observation du détail et des hommes concrets »³. Ensuite:

[1]e moment vint où il se rendit compte que quelque chose, dans ses écrits, n'allait pas, et ce moment fut décisif pour tout le reste de sa vie [...]. II avait voulu atteindre à la pureté du ton moral; mais la pureté, pour être vraie, doit être terrestre, appuyée fortement sur l'expérience et l'observation, autrement elle est fausse. A. avait découvert qu'il s'était embourbé dans la fausseté, parce qu'il se mouvait parmi les idées qu'il se faisait des hommes et non parmi les hommes [...] Il cherchait le moyen de provoquer chez le lecteur la résonance émotionnelle. Évidemment le lecteur, en face de mots tels que la grâce ou le péché, qui lui sont connus dès l'enfance, réagit avec intensité. Mais dans l'abus de ces mots, il y a quelque chose de malhonnête. A. douta de la réalité des conflits qu'il avait créés (p. I22).

D'un côté les yeux mal entraînés et l'abus des mots, de l'autre le mécanisme qui est au cœur du kitsch, la recherche de la résonance émotionnelle comme simple effet. Notons qu'être incapable de s'appuyer sur l'observation et être enclin à abuser des mots ne sont pas des vices éthiques. Dans le

3. L'italique ici et ailleurs est de moi. - KM 
diagnostic d'A., c'est encore un vice non éthique qui explique ses autres vices:

Je crois que la divergence ultérieure de nos destinées était contenue dans le léger écart de nos réactions au moment où nous visitions les ruines de Varsovie ou lorsque nous regardions les prisonniers. Je sentais l'impossibilité où j'étais d'écrire sur ces choses; il m'aurait fallu dire toute la vérité, et non pas une partie [...] Nous étions étrangement gênés, je m'en souviens, lorsque A. nous lisait, dans la ville terrorisée, des récits qui exploitaient ce thème tout chaud [...] Des milliers de personnes mouraient autour de nous dans les tortures. Transformer immédiatement leurs souffrances en tragique théâtral nous paraissait indécent. Il vaut mieux parfois bégayer par excès d'émotion que parler en phrases arrondies. La voix intérieure qui nous arrête, quand il y a trop à exprimer, est sage. Il est possible que A. n'ait pas connu cette voix. Seule la passion pour la vérité aurait pu l'empêcher de devenir ce qu'il devint (p. 147-7).

La passion pour la vérité, et donc l'attachement à la valeur de la vérité, est au cœur d'une vertu qui n'est pas éthique mais cognitive ou intellectuelle. Sans cette passion et son rapport d'opposition dynamique à un certain nombre de tentations familières, A. n'aurait pas incarné le spectacle même de l'irrationalité:

Il n'eût pas écrit, alors, le roman du vieux communiste et de la jeunesse démoralisée; là, il s'était permis la pitié, dans les limites où il le pouvait sans s'exposer à la censure, et il avait quêté l'approbation en simplifiant l'image des événements, en accord avec les désirs du Parti. Un premier compromis en amène un second et un troisième, jusqu'au moment où enfin tout ce qu'on dit est parfaitement rond et cohérent, mais n'a plus rien de commun avec la chair et le sang des hommes. Tel est, dans la dialectique, le revers de la médaille. Pour le confort mental qu'elle donne, il faut payer. Autour d'A. vivaient et vivent beaucoup d'ouvriers et de paysans dont la parole est maladroite; mais en définitive, la voix intérieure qu'ils entendent n'est pas si différente de cette exigence qui souvent ferme la bouche de l'écrivain et lui impose: tout ou rien. Qui sait? Peut-être un paysan inconnu ou un petit facteur des postes doivent-ils être placés plus haut, dans la hiérarchie de ceux qui servent l'humanité, qu'A. le moraliste (p. I47).

A. a quelques-uns des traits du pharisaïsme: il «sut réaliser avec succès son idéal de l'écrivain-autorité-morale» (p. I24), il y a un «...ton prêcheur et hautain, toujours propre à A. » (p. I45). "Ses camarades, enviant à A. les succès qu'il devait à la noblesse de son ton, l'appelaient "la... respectueuse" " (p. I46). Tout comme la passion pour la vérité s'oppose à tous les affirmations et jugements qui nous rendent la vie plus agréable, elle s'oppose au kitsch ainsi qu'à sa contrepartie éthique, le pharisaïsme.

B., "l'amant malheureux», est au contraire à plusieurs égards un héros cognitif: dans tous ses récits, B. est un nihiliste. Par nihilisme, je n'entends pas amoralité. Au contraire, ce nihilisme provient d'une passion éthique, c'est un amour malheureux du monde et des hommes. En décrivant ce 
qu'il a vu, B. veut aller jusqu'au bout, présenter exactement un monde dans lequel il n'y a pas de place pour l'indignation. Le genre humain, dans les contes de B., est $n u$, dépouillé des bons sentiments qui durent autant que dure la coutume de la civilisation.

Sa vertu intellectuelle semble expliquer sa vertu éthique:

B. veut être dur. Il veut regarder froidement et il ne s'épargne pas; il a peur $d u$ mensonge; ce serait un mensonge de se présenter lui même comme un observateur qui juge, tandis qu'en réalité, il était comme tous soumis à toutes les lois de la dégradation. Exagérément honnête [...] (p. I63).

Si B. possède les vertus intellectuelles qui manquent à A., B. est critiqué pour sa vision globale, vision globale dont $\mathrm{A}$. est incapable, mais aussi pour sa cécité par rapport à ce qui est particulier :

B. était un bon observateur, quoique son don d'observation fût tourné avant tout vers l'absurde, la laideur et la méchanceté d'autrui. Il était à vif, irritable et intolérant. Peut-être son amertume aurait-elle été moins forte si, après ces années de souffrance, il avait $p u$ s'arrêter en un point et embrasser du regard, non cette mêlée agitée par le paroxysme marquant la fin de la guerre, mais l'homme particulier (p. 163-4).

La Gesinnung de B., sa haine, l'emporte sur sa passion de vérité pour s'en servir:

À mesure qu'il faisait connaissance avec les œuvres des théoriciens du léninisme-stalinisme, il se laissait convaincre que c'était justement là ce qu'il avait cherché. Cette haine qui existait en lui peut être comparée à un torrent qui détruit tout ce qu'il rencontre. Sa haine (ou son amour malheureux) du monde se précipitait en avant, inutilement. Quoi de plus simple que de diriger son courant dans le sens voulu et même d'y établir de grands moulins qu'il ferait tourner? Quel soulagement! la haine utile, la haine au service de la société!

B., comme l'éponge qui se remplit d'eau, absorbait le «matérialisme dialectique ». Le côté matérialiste de ce système assouvissait son désir de vérité brutale. Le côté dialectique permettait de faire un saut au-dessus du genre humain, de regarder l'humanité comme le matériel de l'histoire (p. I66-7).

La vérité est souvent brutale ou cruelle, comme le rappelle Musil dans L'Homme sans qualités, mais cette brutalité-là est sans commune mesure avec la qualité des vérités et des théories au service de la haine ou du dégoût. Quand la vérité est au service de quelque chose que ce soit il est très facile de céder au kitsch, comme A. et B.:

Le Monde de pierre fut le dernier livre où B. s'efforça d'employer les moyens artistiques reconnus comme efficaces par la littérature occidentale: la retenue, l'ironie cachée, la colère masquée. Il se convainquit vite que tous ces scrupules artistiques ne sont pas nécessaires; au contraire, on l'applaudissait d'autant plus qu'il appuyait plus fortement sur la pédale. À haute voix, violemment, clairement, tendancieusement, voilà comment il devait s'exprimer. Parmi les 
écrivains du Parti (et B. entra au Parti), c'était une surenchère de facilité et de simplification; la limite s'effaçait déjà entre la littérature et la propagande (p. I69).

Les vertus cognitives de B. perdent la bataille contre la conjonction de la vanité, du conformisme et d'un "grain de politique»:

Je l'ai vu, pour la dernière fois, en I950. Depuis le temps qui avait précédé son arrestation par la Gestapo, il avait beaucoup changé. Il n'y avait plus en lui cette réserve et cette fausse humilité d'antan [...]. Le timide poète d'autrefois s'était mué en homo politicus. En cette période, il était déjà l'un des premiers propagandistes. Nul journaliste n'est capable de servir une cause aussi bien qu'un écrivain qui a derrière soi une époque de travail désintéressé. B. usait, dans ses articles venimeux dirigés contre l'Amérique, de toute sa maîtrise du métier. En regardant le visage de ce nibiliste respecté, je me disais que tout l'art d'écrire est une pente savonnée, et que ce n'est qu'avec beaucoup d'efforts qu'on peut éviter de glisser au plus facile. L'impératif intérieur qui suscite ces efforts est plutôt irrationnel: la Nouvelle Foi, qui ne reconnaît pas l'art désintéressé, détruit cet impératif. B., quoiqu'il se refusât à reconnaître aucune exigence de cet ordre, était, dans ses récits du monde concentrationnaire, un véritable écrivain: il ne falsifiait rien, il ne voulait plaire à personne. Plus tard, il introduisit un grain de politique, et, comme une solution sursaturée se cristallise, ce grain a suffi pour changer tout ce qu'il écrivait en quelque chose d'unilatéral et de figé [...]. La différence essentielle entre les grands écrivains [Swift, Stendhal et Tolstoï] qui critiquaient les institutions politiques de leur temps et des gens du type de B. consiste, semble-t-il, en un non-conformisme total chez les premiers: ils agissaient contre leur propre entourage, tandis que B. et ses collègues [...] entendaient déjà les applaudissements (p. I70).

La vanité et le conformisme peuvent suffire à immoler la raison. Mais un moyen plus efficace encore est un certain type de colère:

[B.] subissait une tentation fréquente chez les intellectuels de l'Est, celle d'immoler sa raison. Le processus psychique qui se manifeste chez eux quand ils prennent la plume est assez complexe. Supposons qu'il s'agisse de présenter quelque «événement de politique internationale ». L'interdépendance des phénomènes leur apparaît assez clairement [...] Pour les présenter honnêtement, il faudrait pénétrer les motifs des parties en lutte, les nécessités auxquelles elles sont soumises, en tout cas procéder à une analyse fouillée. Alors c'est la colère qui vient à la rescousse; la colère introduit un ordre immédiat dans le labyrinthe compliqué des corrélations; la colère dispense d'analyser (p. I7I).

La colère en question est celle qui se met au service d'une forme d'autoduperie qui crée l'impression de la vertu épistémique:

C'est une colère dirigée contre l'idée illusoire que quelque chose dépend de la volonté humaine; il s'y ajoute la peur d'être dupe de sa propre naïveté. Puisque le monde est brutal, il faut tout réduire aux éléments les plus simples et les plus brutaux. L'auteur comprend que cette vue n'est pas exacte: la stupidité et les décisions humaines agissent sur les événements non moins que ne font les 
nécessités économiques. Mais en accomplissant un acte de vengeance sur les intentions humaines, en démontrant qu'elles sont complètement déterminées par quelque loi élémentaire, l'auteur ressent sa supériorité: il se considère comme perspicace et assez fort pour vivre sans "préjugés idéalistes» (p. 172).

Cette colère, mobilisée par la haine et son mouvement de détraction toujours plus forte, conduit à cette forme de cécité, qui a déjà été mentionnée, la cécité face à tout ce qui est particulier:

Simplifier, dépouiller les illusions, présenter toute chose dans sa nudité, cette tendance était chez lui constamment visible. Pourtant, quand on va de plus en plus loin vers la nudité du monde, on parvient au point où l'intellect n'a plus rien à dire. Le mot se change en un cri de bataille et il n'est plus qu'un substitut imparfait de l'action [...] Il ne pouvait jamais regarder ni analyser sans haine; mais le mouvement qui l'entraînait était un mouvement accéléré: plus vite, toujours plus vite, toujours plus de vitesse, toujours plus de haine. Les formes du monde devenaient pour lui de plus en plus simples, jusqu'au moment où l'arbre particulier, l'homme particulier cessaient d'avoir une importance quelconque, et où $\mathrm{B}$. se trouvait, non parmi les êtres et les choses, mais parmi les concepts politiques (p. I72).

Ces composantes des vies d'A. et de B. sont sans doute quelques-unes des formes qui peuvent constituer l'esclavage intérieur. Mais qu'ont-elles en commun? La haine, la colère, la vanité, le confort mental et leur conflit avec le devoir sont les fondements ou les points de départ de bon nombre d'analyses de la moralité et de l'éthique. Mais il ne s'agit pas en premier lieu de cela ici. Les processus analysés par notre penseur polonais ne sont pas directement des vices moraux ou éthiques. La haine, la colère et la vanité engendrent ici des vices non éthiques, des vices intellectuels et épistémiques - l'incapacité de voir, la simplification, la préférence pour des vérités partielles quand la vérité totale s'impose, l'adaptation des faits à une thèse préconçue, l'auto-duperie, la mort d'une passion quelconque pour la vérité, l'immolation de la raison.

Le nom de ces vices est la sottise (stultitia, Torheit, foolishness) ${ }^{4}$. Au cœur de chaque vertu, il y a un attachement à une valeur. L'homme juste attache de l'importance à la justice. Au cœur de la sottise il y a une indifférence ou même une hostilité aux valeurs de la connaissance et de la vérité, de la précision, de la vue juste. La sottise n'est pas la stupidité même s'il arrive souvent que les deux termes soient utilisés comme des synonymes. La stupidité est un trait en grande partie inné, mesuré par les tests de QI. À la diffé-

4. Je préfère traduire en français Stultitia, Torheit, Foolishness par sottise et non pas par bêtise, car seul le mot sottise a en français classique le sens visé ici, d'une insensibilité aux valeurs, et non pas seulement un défaut du jugement ou de l'entendement. Cf sur cette distinction P. Engel, Les lois de l'esprit, Paris, Ithaque, $20 \mathrm{I} 2$, p. 177 et p. 246 sq. 
rence de la sottise, elle n'est donc pas un vice. A. et B. ne sont pas stupides. La sottise et l'intelligence sont malheureusement tout à fait compatibles ${ }^{5}$.

La forme particulière de la captivité de l'esprit analysée par notre poète est celle de la sottise. La sottise n'est pas l'étroitesse d'esprit, le dogmatisme, la bigoterie, la contagion affective ni le désir mimétique, autant de phénomènes qui sont eux aussi incompatibles avec la liberté intérieure. Il ne serait pas trop difficile de montrer que dans les analyses de C. - de son «manque d'exactitude» (p. 2I6) - , et de D. — ce «volontaire de l'enthousiasme» (p. 23 I, cf. p. 237), notre poète présente encore des formes de la sottise et de l'absence de liberté intérieure.

Les deux figures majeures du livre, le Ketman et la pilule de Murti-Bing (une figure empruntée à Witkiewicz), sont au cœur des descriptions inoubliables de plusieurs formes de sottise:

Murti-Bing était un philosophe mongol qui aurait trouvé le moyen de transmettre sa vision du monde par une voie organique [...] L'homme qui en fait usage se transforme; il trouve la sérénité et le bonheur [...] Celui qui avale des pilules Murti-Bing est immunisé contre toute préoccupation métaphysique (p. 25).

Parmi les nombreux motifs qui peuvent pousser quelqu'un à prendre cette pilule, il y a "la peur de penser par soi-même» (p. 32). La figure de la pilule de Murti-Bing n'est pas sans rappeler une expérience de pensée célèbre du philosophe américain Robert Nozick. Selon une variante de cette expérience, on vous invite à entrer dans une "machine à plaisir ". On vous explique, avec force arguments solides et fiables, qu'une fois à l'intérieur de la machine vous ne connaîtrez que le plaisir, la sérénité, le bonheur. Vos émotions exclusivement positives seront des réactions à des croyances, expériences et souvenirs d'une vie riche en amitiés, réussites et activités, en réalisations de valeurs positives - d'une vie qui ressemble en tous points à celle dont vous rêvez. Mais toutes ces croyances, ajoute-t-on, seront erronées, vos expériences illusoires, vos souvenirs des faux souvenirs. Acceptezvous d'y entrer? On peut penser qu'un seul motif pourrait amener quelqu'un à ne pas y entrer: le rejet des croyances erronées et des illusions, une conscience vive de leur valeur négative, et, par conséquent, un rejet des émotions qui colorent de telles croyances et de telles illusions de la valeur négative des «fausses » émotions. Il y a pourtant une autre réponse à l'invitation. Selon le postmoderniste on ne peut pas distinguer entre les croyances vraies et les croyances fausses, entre les perceptions et les illusions. Un postmoderniste qui se respecte ne dit pas d'une affirmation qu'elle est vraie ou fausse. Elle est, dit-il, au plus «vraie». Les guillemets, écrits ou oraux, signalent sa 
méfiance de la vérité et donc de la valeur présumée de celle-ci. Il se méfie également de la justification, de la clarté, de l'objectivité. Il est sot.

La figure du Ketman (empruntée pour sa part à Gobineau) est celle d'un mode de vie qui est caractérisé par des formes extrêmes de ce que l'on appelait autrefois l'accommodation ${ }^{6}$. On se rallie à des avis que l'on ne partage pas. On s'efforce de garder une liberté intérieure. Ce qui distingue le Ketman de l'accommodation ordinaire, c'est d'abord le fait qu'il s'agit d'une «institution permanente» (p. 87) et ensuite de ses effets:

[C]ette mascarade continuelle [...] assure [...] aux hommes masqués des satisfactions appréciables $[\ldots][\mathrm{O}] \mathrm{n}$ se met à apprécier par-dessus tout sa propre ruse. Le succès dans le jeu devient alors une source de joie. Le moi, qui est protégé contre le regard des curieux, acquiert une valeur toute spéciale [...] (p. 86).

La personne intime (ou le moi intime) se cache derrière la personne sociale (ou le moi social) ou plutôt derrière plusieurs personnes sociales (ou moi sociaux). Car «le nombre de variétés de Ketman est presque illimité " (p. 90). Il y a le Ketman «national», celui de la pureté révolutionnaire, le Ketman esthétique, professionnel, sceptique, métaphysique, éthique. La place occupée par le jeu est telle qu'elle modifie considérablement la personne intime (ou le moi intime). «Le Ketman, dit Gobineau, enorgueillit celui qui le met en pratique» (p. 87).

Celui qui s'adonne au Ketman doit mentir. Mais serait-il moins faux s'il pouvait dire la vérité (p. II3)?

Le Ketman repose [...] sur la réalisation du soi contre l'obstacle [...] si cette résistance s'évanouissait soudain, il se trouverait dans le vide [...] (p. II3).

Qui sait si l'absence d'un centre intérieur, chez l'homme, n'explique pas le mystère du succès de la Nouvelle Foi [...] La Nouvelle Foi, en soumettant l'homme à une pression puissante [...], fait naître le sentiment que ce centre existe. La peur devant la liberté n'est rien d'autre que la peur devant le vide [...] Considérant qu'en lui il n'y a rien, l'homme accepte aujourd'hui n'importe quoi, même s'il sait que ce "n'importe quoi» est mauvais, pourvu qu'il se trouve avec les autres et ne soit pas seul (p. II 4$)^{7}$.

Pour comprendre toute la portée de la philosophie de la sottise et de la captivité de l'esprit chez notre penseur, il convient de considérer un texte remarquable de 1942-1943, dont je ne connais que la traduction anglaise, "The Legend of the Will», et qui est consacré à une analyse de Julien Sorel. Julien serait un homme de ressentiment:

His personality is suffused with ressentiment, that complex feeling composed of envy, anger, longing for what is beyond his reach, and an artificially created

6. Cf. Morley, I923. Les doctrines traditionnelles d'une «double vérité » sont des analyses de l'accommodation.

7. Christopher Hitchens (20I I, p. 619) prolonge l'analyse de Miłosz et distingue différentes formes du Ketman dans l'Iran d'aujourd'hui. N'y a-t-il pas aussi un Ketman de la rectitude politique (political correctness), le dernier avatar du pharisaïsme? 
contempt for the values he holds in secret from himself, but which he cannot attain.

In The Red and the Black, Stendhal gave a living form to the figure we have been speaking of [...]. There is just a single hero, and the entire plot is filled with his struggle against society or, to be more precise - and this is essential for ressentiment - against an image of society that is reflected in his psyche, filled as it is with grievances.

Pour l'analyse du ressentiment Miłosz renvoie à la traduction française de la monographie de Max Scheler ${ }^{8}$ sur les raisins verts, une monographie consacrée à un développement de l'analyse célèbre de Nietzsche (Généalogie de la morale), à une multiplicité d'exemples de ressentiment et à une critique de ce que dit Nietzsche au sujet de son exemple préféré de ressentiment, la psychologie du christianisme.

Le Rouge et le Noir a été l'objet d'un grand nombre d'analyses pénétrantes (Alain, Roger Bauer, Léon Blum, Victor Brombert, Jon Elster, Patrizia Lombardo, etc.). Mais c’est surtout et d'abord Miłosz qui a compris le cœur de Julien'. S'il a un prédécesseur, c'est peut-être Nietzsche dont on connaît l'admiration qu'il portait à Stendhal.

Qu'est-ce donc que le ressentiment? Au cœur des réponses données par Nietzche et Scheler se trouve la thèse selon laquelle la femme du ressentiment est amenée à changer ses évaluations, à juger mauvais (bon) ce qu'elle avait su auparavant être bon (mauvais). Comme dans la fable, le jugement correct selon lequel les raisins sont bons est remplacé par le jugement selon lequel ils sont mauvais (verts). Pourquoi la femme du ressentiment est-elle amenée à une telle réévaluation? Parce qu'il lui est trop difficile de vivre avec la vérité et avec la connaissance, parce que la réévaluation lui rend la vie plus agréable, diminue sa conscience de sa propre impotence. Dans ce processus d'auto-duperie la faiblesse, l'impotence, l'envie, la haine et la répétition des affects négatifs joueraient des rôles variables.

Cette esquisse simplifie quelque peu la pensée de Nietzsche, qui semble souvent vouloir nier aux évaluations la possibilité d'être vraies ou fausses ${ }^{10}$. L'analyse est aussi erronée sur au moins un point. Elle n'est pas suffisamment générale. Certes, la transition d'un jugement évaluatif vrai (d'une connaissance axiologique) à un jugement évaluatif faux, motivée exclusivement par le confort qu'elle procure, constitue un exemple d'irrationalité et de sottise , d'indifférence aux valeurs de la connaissance et de la vérité. Mais la transition d'un jugement évaluatif faux à un jugement vrai (ou faux) motivée de la même façon est, elle aussi, un exemple de sottise.

8. Scheler I933, une traduction de Scheler 1955.

9. Cf. aussi Baumann I964, p. I3, p. 30.

10. Ce n'est pas le cas de Miłosz; cf. Miłosz I988, p. 274. 
Julien est à plusieurs égards un héros cognitif. Comme d'autres héros et héroïnes stendhaliens et comme le narrateur beylien, il s'interroge souvent sur les motifs des réactions affectives. Mais sa vertu épistémique a des limites:

[A] reversal of the hierarchy of values takes place in him; he labors unceasingly at discovering the true (or so he believes) springs of all noble deeds and virtues, assigning to them the significance of a means of struggle for existence and depriving them of their independent meaning. Exaltation at his own perceptiveness and strength follows upon this. He alone sees things as they are; he alone is not deceived, whereas the rest, wear the yoke of stupid, purely conventional laws and commandments .

Sorel is a titan of the will. "Woe to him who stands out." To conceal in his heart his hatred, his faith in his own difference, his superiority, and to behave like everyone else, but consciously, to lie even about the most minute details, this is his commandment. A dreadful battle rages in his heart, a battle against weakness, against each of its manifestations. Love, devotion, trust, faith are all weaknesses. Delight, pleasure, relaxation, gaiety, are all weaknesses. He is an ascetic of the will, a connoisseur of a stern ethics, the ethics of one's duty to oneself. Until finally his will loses the goal it was supposed to serve and becomes a goal in itself, and exercising it becomes the highest commandment.

Julien a son Ketman à lui et ressemble quelque peu à B. Il se flatte d'être un héros cognitif. Mais ses capacités cognitives sont subordonnées à sa volonté de puissance, de s'imposer contre la société environnante. Cette subordination et le plaisir qu'il prend dans l'exercice de sa volonté forte faussent ses perceptions. La volonté de puissance de Julien, de plus en plus émancipée de toute vertu cognitive, anticipe la volonté de puissance de Nietzsche ainsi que les rhétoriques décisionnistes de Heidegger et de Sartre (lui aussi, comme Nietzsche, un beylien). Il s'agit de différentes formes de ce que Miłosz appelle - en I942-1943 - «la déification de la volonté », une déification que seule une poignée de penseurs - Scheler, Klages, Musil vont essayer de démystifier:

In all these cases, we are dealing with the deification of the will, and in all of these cases, an obsession with necessity is concealed at their base: Living within the social monster, I am always aware of the necessity of my position. I am constantly dependent on other people; I rub up against them; I am tumbled about like a stone in a rushing stream. Stifled revulsion explodes into rebellion against necessity, seeks out the illusion, at least, of freedom. It is not the fact of doing battle itself that is essential for these characters, but rather that in the course of things, the battle loses any utilitarian justification. It is transformed into a struggle with the phantoms of one's own imagination; the one who does battle sees in his own nature the elements he must smother because they are the collaborators with the social monster, the forward guard deployed by a collective means of sensibility in order to attack the strong man from within. "Human" feelings like pity, empathy, or kindness appear to be dangerous drugs. 
Mais si le ressentiment et donc la sottise de Julien l'obligent à nier une valeur positive au cœur humain,

[n]onetheless, all of Stendhal's sympathy and all of the reader's sympathy are on the side of the hero. He represents a higher, richer, more perfect type. There is no human sensation that is alien to him. He is capable of loving intensely and truly, of weeping out of pity and hatred for the wrongdoer when he hears a song about prison. He is capable of praying, of feeling remorse for his sins, even though he thinks of religion as a collection of sophistic formulas, one of the countless lies told by human beings.

La perversion des vertus cognitives de Julien, qui est la conséquence de sa volonté de puissance, l'amène à n'attacher aucune valeur à ses états affectifs ni à leurs objets:

But let us be clear: all these are states that are no more meaningful than physiological states. He towers above them; the states change, but he remains unchanging. He is a subject who takes on various selves in turn, but he continues, independently, alongside them. No belief, no thought contains him; he is aware that both beliefs and thoughts flow through him; he pays attention to them in the way one pays attention to one's own pulse, to the beating of one's heart. He is beyond good and evil, beyond truth and falsehood. The one thing that is worthy and of value is to observe oneself and others clearly, not to deceive oneself.

Julien irait même au-delà d'une simple réévaluation des valeurs:

Overturning the hierarchy of values is not a matter of calling things bad that have been considered good until now, or bad things good. Here, the very life force is opposed to all moral values; they become secondary, of little import.

$\mathrm{Si}$, comme le dit très bien Miłosz,

Nietzsche crying out, "Pereat veritas, fiat vita" — let truth die, let life begin - cast a spell that explains nearly the entire intellectual ferment of the past century (p. 46).

(Musil parle plus généralement des «adaptations» du Geist à la vie), c'est bien Julien qui préfigure cette formule magique.

Trois des contextes auxquels appartiennent les analyses de Miłosz méritent d'être rappelés, ne serait-ce que parce que deux d'entre eux sont à peu près inconnus et largement absents des histoires intellectuelles reçues.

Il y a d'abord l'extraordinaire tradition de philosophie et de logique polonaise inaugurée par Twardowski, qui, pénétré de l'idéal de la philosophie scientifique de son maître viennois, Franz Brentano, a transmis cet idéal à plusieurs générations de penseurs qui constituent la gloire de l'École de Lvov-Varsovie: Lukasiewicz, Lesniewski, Jaskowski, Tarski, Ajdukiewicz, etc. ${ }^{11}$ L'importance suprême dans cette tradition des valeurs épistémiques de

11. Cf. Wolenski 2012. 
la clarté et de la précision ainsi que l'horreur du bavardage sont sans doute en partie responsables de ses réussites théoriques remarquables. Le roman de Stanislaw Ignacy Witkiewicz, Insatiabilitée ${ }^{12}$, le point de départ des analyses de La pensée captive, communique ici et là et exemplifie l'atmosphère intellectuelle de cette obsession polonaise pour la clarté. Cette obsession ne se limite pas à l'École de Lvov-Varsovie: elle imprègne, par exemple, les travaux du phénoménologue polonais, Roman Ingarden, lui aussi héritier de la tradition brentanienne, ainsi que ceux du grand philosophe du droit, Léon Petrasizky. J'ignore le degré de connaissance qu'avait Miłosz de ces traditions intellectuelles. Mais il est certain qu'aux États-Unis Tarski appréciait la vodka faite maison de son ami Miłosz (communication personnelle de Jan Wolenski).

La pensée captive appartient ensuite à une série d'écrits publiés entre la fin des années vingt et le début des années cinquante, dont le but est d'analyser ce qui se passe quand les valeurs cognitives sont subordonnées à d'autres valeurs ou rejetées, selon l'analyse de ce qui a été appelé ici la sottise. Leurs auteurs sont José Ortega y Gasset, Ernst Robert Curtius, Bertrand Russell, Robert Musil, Julien Benda ${ }^{13}$, Louis Rougier, Raymond Aron et Josef Schumpeter.

Enfin, comme l'on sait, La pensée captive fait partie de la série de tentatives d'Arthur Koestler, George Orwell, Raymond Aron et d'Eric Hoffer, entre autres, de comprendre le fonctionnement de l'esprit dans les démocraties populaires et les États totalitaires. Selon le jugement de l'historien de l'Europe au vingtième siècle, Tony Judt, le livre de Miłosz "is by far the most insightful and enduring account of the attraction of intellectuals to Stalinism and, more generally, of the appeal of authority and authoritarianism to the intelligentsia $\gg^{14}$.

Aujourd'hui, la compréhension de la sottise est loin des préoccupations des penseurs, des «intellectuels » et du public. (La stupidité par contre est l'objet de nombreuses recherches.) À la place de l'immense intérêt que portait un Musil ou un Benda à la philosophie de la sottise, on trouve aujourd'hui, partout, l'éthique. Au sein de systèmes universitaires aux moyens de plus en plus restreints, on n'a de cesse de créer des chaires d'éthique. Le mot «éthique» est sur toutes les lèvres. Il y a une mode éthique, de l' «ethical banking», et on boit du café éthique. Comme par hasard, la montée de l'éthique coïncide avec la montée de la rectitude politique. À quand un rec-

12. Roman traduit en français par Alain Van Crugten sous le titre L'inassouvissement (Lausanne, L'Âge d'homme, I970).

13. Sur Benda, cf. Walzer I988, chap. 2, et surtout Engel 20I2, chap. 4: «Le clerc et les valeurs intellectuelles ", in Les lois de l'esprit, Julien Benda ou la raison, Paris, Ithaque, 20I 2, chap. 3 .

14. Judt 20I I, p. I75. 
teur sage qui remplacera ne serait-ce qu'une seule chaire d' «éthique appliquée » par une chaire pour la compréhension de la sottise?

Jaroslaw Iwaszkiewicz rapporte un avis de Sartre au sujet de La pensée captive: "It's not enough to be smart, you also have to have sagess $\mathrm{e}^{15}$." La sagesse s'oppose traditionnellement à la sottise. Mais les philosophies traditionnelles de la sagesse pêchent sur au moins deux points. Elles donnent un contenu positif très riche à la sagesse, en particulier elles y voient non seulement la vertu intellectuelle, mais certains types de savoir très précis au sujet des choses ultimes, de Dieu. On peut penser par contre que la sagesse s'oppose à la sottise en ceci que la sagesse est l'absence de sottise et que son seul contenu positif est la connaissance, et la conscience vive de la valeur de la connaissance, de la vérité et de ce qui en découle. La structure du rapport entre la sagesse et la sottise serait donc analogue à celle qui, selon tant de traditions de philosophie politique libérales et conservatrices relie la justice à l'injustice: la justice n'a pas de contenu positif, elle est l'absence de différents types d'injustice.

S'il est vrai que dans le domaine politique Sartre avait tort sur à peu près tout (et Aron raison), cela ne veut pas dire que Sartre n'était pas «smart». Mais Sartre et les siens (par exemple Paul Nizan) n'étaient-ils pas sots et Miłosz sage?

\section{Références}

Baumann, C. Literatur und intellektueller Kitsch. Das Beispiel Stendhals. Zur Sozialneurose der Moderne, Heidelberg, Wolfgang Rothe Verlag, I964.

Engel, P. Les lois de l'esprit, Julien Benda ou la raison, Paris, Ithaque, $20 \mathrm{I} 2$.

Gomperz, H. (1904) Die Lebensauffassung der griechischen Philosophen und das Ideal der inneren Freiheit, Jena, Eugen Diederichs, I9I 5.

Hadot, P. Qu'est-ce que la philosophie antique? Paris, Gallimard, 1995.

. Exercices spirituels et philosophie antique, nouvelle éd., Paris, Albin Michel, Bibliothèque de l'évolution de l'humanité, 2002.

Hitchens, C. Arguably, New York, Twelve, Hachette, 20 I I.

Judt, Tony. The Memory Chalet, Londres, Vintage Books, 20 I I.

Krieger, Leonard. The German Idea of Freedom, University of Chicago Press, 1957. Miłosz, Czesław (1953) (1988). La pensée captive. Essai sur les logocraties populaires, Paris, Gallimard, I994.

- A Year of the Hunter, Farrar-Straus-Giroux, New York, I996. and Letters from Occupied Poland, 1942-1943, New York, Farrar Straus Giroux, p. 33-48.

Morley, J. On Compromise, London, Macmillan, I923.

15. Miłosz I994, p. I 46. Adam Zagajewski a cité ce mot sartréen lors d' un colloque au Château de Coppet. Jan Wolenski a trouvé la référence. Je les en remercie. Je remercie également Olivier Massin pour ses conseils linguistiques et philosophiques. 
Mulligan, K. "Ironie, valeurs cognitives et bêtise », Philosophiques, vol. 35, $\mathrm{n}^{\circ} \mathrm{I}$, Les valeurs de l'ironie, Pascal Engel (dir.), 2008, p. 89-I07. http://www.unige.ch/lettres/philo/enseignants/km/doc/ironie_betise.pdf . "Torheit, Vernünftigkeit und der Wert des Wissens", Wissen und Werte, G. Schönrich (dir.), Paderborn, mentis Verlag, 2009, p. 27-44. http://www.unige.ch/lettres/philo/enseignants/km/doc/FoolTorheit4.pdf

Scheler, M. L'homme du ressentiment, Paris, Gallimard, I933.

- (I9I2, I9I 5) "Das Ressentiment im Aufbau der Moralen", Vom Umsturz der Werte. Abhandlungen und Aufsätze, Gesammelte Werke, III, Berne, Francke Verlag, I955, p. 33-I47.

. "Zur Psychologie des englischen Ethos und des cant», Politisch-pädagogische Schriften, Gesammelte Werke, IV, Berne, Francke Verlag, I982, p. 2 I 8250.

Voelke, A. J. La philosophie comme thérapie de l'âme, Fribourg-Paris, I993.

Walzer, M. The Company of Critics. Social Criticism and Political Commitment in the Twentieth Century, New York, Basic Books (trad. fr. Le deuxième âge de la critique sociale, Paris, Métailié, 1996), I988.

Wildauer, T. Die Psychologie des Willens bei Sokrates, Platon und Aristoteles. I. Theil: Sokrates Lehre vom Willen, Innsbruck, Verlag der Wagner'schen Universitäts-Buchhandlung, I877.

Wolenski, J. L'école de Lvov-Varsovie. Philosophie et logique en Pologne (I895I939), Paris, Vrin, 2012. 